\title{
Use of glecaprevir/pibrentasvir in patients with chronic hepatitis $C$ virus infection and severe renal impairment
}

\author{
Desmond Y. H. Yap', Kevin S. H. Liư ${ }^{2}$, Yu-Chun Hsu ${ }^{3}$, Grace L. H. Wong ${ }^{4}$, Ming-Chang Tsai ${ }^{5}$, Chien-Hung Chen ${ }^{6}$, \\ Ching-Sheng Hsu', Yee Tak Hui ${ }^{8}$, Michael K. K. Li ${ }^{9}$, Chen-Hua Liu ${ }^{10}$, Yee-Man Kan ${ }^{11}$, Ming-Lung Yu ${ }^{12}$, and \\ Man-Fung Yuen ${ }^{2}$
}

'Division of Nephrology, Department of Medicine, Queen Mary Hospital, The University of Hong Kong, Hong Kong; '²ivision of Gastroenterology and Hepatology, Department of Medicine, Queen Mary Hospital, The University of Hong Kong, Hong Kong, China; ${ }^{3}$ Division of Gastroenterology, Department of Internal Medicine, Changhua Christian Hospital, Taiwan; ${ }^{4}$ Department of Medicine and Therapeutics, State Key Laboratory of Digestive Disease, Institute of Digestive Disease, Prince of Wales Hospital, The Chinese University of Hong Kong, Hong Kong, China; 'Division of Gastroenterology, Department of Internal Medicine, Chung Shan Medical University Hospital, Taichung; ${ }^{6}$ Department of Internal Medicine, National Taiwan University Hospital Yunlin Branch, Douliu; ${ }^{7}$ Liver Diseases Research Centre, Taipei Tzu Chi Hospital, Buddhist Tzu Chi Medical Foundation, Taipei, Taiwan; ${ }^{8}$ Department of Medicine, Queen Elizabeth Hospital, Hong Kong; ${ }^{9}$ Department of Medicine and Geriatrics, Tuen Mun Hospital, Hong Kong, China; ${ }^{10}$ Department of Internal Medicine, National Taiwan University Hospital, Taipei, Taiwan; "Department of Medicine and Geriatrics, Kwong Wah Hospital, Hong Kong, China; ${ }^{12}$ Division of Hepatobiliary, Department of Internal Medicine and Hepatitis Centre, Kaohsiung Medical University Hospital, Kaohshiung Medical University, Kaohsiung, Taiwan

\section{Graphical Abstract}

Use of glecaprevir/pibrentasvir in patients with chronic hepatitis C virus infection and severe renal impairment

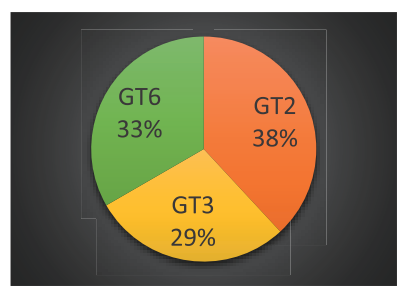

- Twenty-one patients with chronic hepatitis $\mathrm{C}$ virus (HCV) (GT2 n=7, GT3 n=6, GT6 n=8) infection and severe renal impairment received Glecaprevir/pibrentasvir (G/P) GLE/PIB for $11.2 \pm 1.8$ weeks

- 16 patients received $\mathrm{G} / \mathrm{P}$ during dialysis (7 on peritoneal dialysis and 9 on haemodialysis)

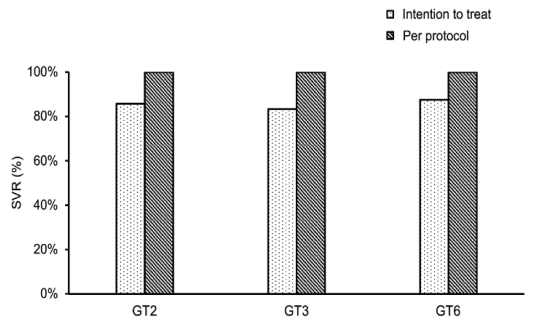

- One patient died of dialysis-related peritonitis and two were lost to follow-up

- The SVR12 rate in the remaining 18 patients was $100 \%$

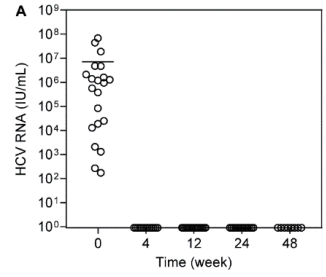

All patients achieved undetectable levels at 4-, 12-, 24- and 48-weeks after treatment.

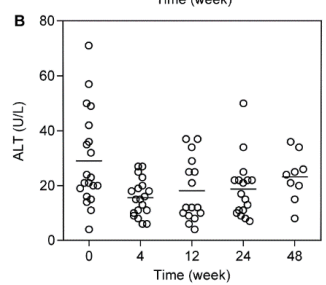

Patients with deranged ALT showed normalization after 4 weeks and the response was sustained for $\mathbf{4 8}$ weeks.

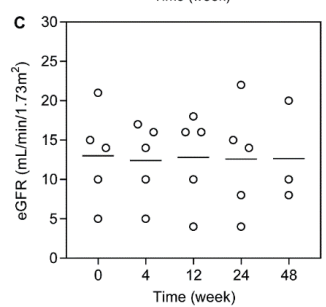

Renal function remained stable for those not yet on dialysis

GLE/PIB treatment was associated with high efficacy and tolerability in HCV-infected patients with severe renal impairment 
Background/Aims: Data on treatment efficacy and safety of glecaprevir/pibrentasvir (GLE/PIB) for chronic hepatitis C virus $(\mathrm{HCV})$ infection in Asian patients with severe renal impairment are limited. This study aimed to study the treatment and side effects of GLE/PIB in these patients infected with non-1 genotype (GT) HCV.

Methods: We prospectively recruited patients with Child's A cirrhosis and eGFR $<30 \mathrm{~mL} / \mathrm{min} / 1.73 \mathrm{~m}^{2}$ in Hong Kong and Taiwan during 2017-2018 to receive GLE/PIB treatment.

Results: Twenty-one patients (GT2, $n=7 ; \mathrm{GT3}, \mathrm{n}=6$; and GT6, $\mathrm{n}=8$ ) received GLE/PIB for $11.2 \pm 1.8$ weeks. All except one were treatment-naïve. GLE/PIB was initiated in 16 patients while on dialysis (seven on peritoneal dialysis [PD] and nine on hemodialysis) and in five patients before dialysis. One patient died of PD-related peritonitis during treatment and two were lost to follow up. The SVR12 rate in the remaining 18 patients was 100\%. All patients achieved undetectable levels at 4-, 12-, 24- and 48-week after treatment. Patients with deranged alanine aminotransferase showed normalization after 4 weeks and the response was sustained for 48 weeks. No significant adverse event was observed.

Conclusions: GLE/PIB treatment was associated with high efficacy and tolerability in HCV-infected patients with severe renal impairment. (Clin Mol Hepatol 2020;26:554-561)

Keywords: Glecaprevir and pibrentasvir; Hepatitis C; Renal insufficiency

\section{Study Highlights}

1. Glecaprevir and pibrentasvir treatment is associated with high SVR in patients with non-genotype $1 \mathrm{HCV}$ infection and severe renal impairment.

2. Glecaprevir and pibrentasvir treatment is safe and well-tolerated in patients with severe renal impairment, including those on hemodialysis and peritoneal dialysis.

\section{INTRODUCTION}

Chronic hepatitis C virus (HCV) infection is an important cause of cirrhosis and hepatocellular carcinoma (HCC), and confers detrimental effects on the survival of patients undergoing regular dialysis and renal transplantation. ${ }^{1-3}$ The conventional treatment for chronic HCV infection is interferon-based therapy, with or without the combination of ribavirin. Such regimens had suboptimal therapeutic efficacy in patients with advanced chronic kidney disease (CKD), and are often associated with substantial side effects which lead to treatment discontinuation. ${ }^{4}$ The use of direct acting antiviral (DAA) have revolutionized the management of chronic HCV infection. In the general population, DAA-based treatments (with or without ribavirin) are associated with excellent efficacy and tolerability in HCV patients with or without cirrhosis, ${ }^{5-7}$ and yet clinical data on their use in patients with severe renal insufficiency remains relatively limited. ${ }^{1,8-11}$

Glecaprevir/pibrentasvir (GLE/PIB) is a NS3/4A protease inhibitor/NS5A inhibitor combination drug which shows high anti-viral efficacy across six major genotypes (GT) of HCV and favourable pharmacokinetic properties in renal failure patients (once-daily oral dosing and with negligible $(<1 \%)$ renal excretion). ${ }^{12-18}$ The use of GLE/PIB without concomitant administration of ribavirin is another merit for its application in patients with advanced CKD. ${ }^{19}$ The data from EXPEDITION-IV suggested that GLE/PIB treatment was associated with good virological response and safety profile in patients with severe renal impairment. ${ }^{19}$ Although this study reported that GLE/PIB was effective across all six major GT in patients with significant renal dysfunction, one should note that patients included mostly suffer from GT1 ( 52\%) and there was only

\section{Abbreviations:}

AFP, alpha-fetoprotein; ALT, alanine aminotransferase; CKD, chronic kidney disease; DAA, direct acting antiviral; GFR, glomerular filtration rate; GLE, glecaprevir; GT, genotype; HCC, hepatocellular carcinoma; HCV, hepatitis C virus; HD, hemodialysis; IFN, interferon; PD, peritoneal dialysis; PIB, pibrentasvir

\section{Corresponding author: Man-Fung Yuen}

Department of Medicine, The University of Hong Kong, Queen Mary Hospital, 102 Pokfulam Road, Hong Kong, China

Tel: +852-22553994, Fax: +852-28162863

E-mail:mfyuen@hku.hk

https://orcid.org/0000-0001-7985-7725 


\section{CLINICAL and MOLECULAR}

\section{HEPATOLOGY}

one patient (1\%) with GT6 HCV infection. In addition, only 9\% of the study patients were of Asian origin. In this context, GT6 HCV infection is more prevalent in Southern China, Hong Kong, Taiwan and other Southeast Asian countries, ${ }^{20,21}$ and data for GLE/PIB treatment for non-1 GT (especially GT6) HCV infection in patients with severe renal impairment renal is lacking.

\section{PATIENTS AND METHODS}

\section{Patients}

We prospectively recruited patients with chronic HCV infection and severe renal impairment to receive GLE/PIB treatment. The inclusion criteria were: 1) patients who were at least 18 years of age and infected with $\mathrm{HCV}$; 2) patients with $\mathrm{GT2}$, GT3, GT5, or GT6 HCV infection, and compensated cirrhosis (Child-Pugh score S6) and/or extra-hepatic manifestations of HCV infection; and 3) CKD of Stage 4 (eGFR $15-29 \mathrm{~mL} / \mathrm{min} / 1.73 \mathrm{~m}^{2}$ ) or Stage 5 (eGFR $<15 \mathrm{~mL} / \mathrm{min} / 1.73 \mathrm{~m}^{2}$ or requiring dialysis or kidney transplantation). Exclusion criteria were: 1) co-infection with chronic hepatitis B virus; 2) Child-Pugh B or C cirrhosis or history of hepatic decompensation (e.g., bleeding varices, encephalopathy, refractory ascites); 3) prior treatment with an NS5A or protease inhibitor; 4) life expectancy of less than 1 year; 5) patients with unknown HCV genotype; 6) patients with neoplasm, other than HCC, requiring chemotherapy during DAA treatment; 7) patients with newly diagnosed HCC or recurrent HCC within the past 12 months; 8) patients who required treatment with any of the following during DAA therapy, and could not be changed to an alternative medication: dabigatran, atazanvir, efavirenz, lopinavir, carbamazepine, rifampicin, atorvastatin, lovastatin, simvastatin, ethinylestradiol contraceptives and hormone replacement therapies, St. John's wort; and 9) patients who were pregnant. All patients underwent ultrasonography and transient elastography (Fibroscan ${ }^{\circledR}$; Echosens France, Paris, France) to document evidence of liver cirrhosis ( $\geq F 4$ by Fibroscan ${ }^{\circledR}$ [Echosens France] and evidence of portal hypertension on ultrasonography). Fibroscan ${ }^{\circledR}$ (Echosens France) was performed in hemodialysis (HD) patients after a HD session and after peritoneal dialysate had been fully drained out in peritoneal dialysis (PD) patients. In patients who did not have fibroscan because of no reimbursement, the diagnosis of cirrhosis was based on compatible clinical/ultrasonographic features. The study was approved by the Institutional Review Board of the University of Hong Kong/Hospital Authority Hong Kong West Cluster (Approval No.
UW-18-370) and ethics committees of all participating centres.

\section{Use of GLE/PIB treatment and follow-up schedule}

Patient demographics, complete blood counts, liver and renal biochemistry, HCV GT and RNA levels and serum alpha-fetoprotein (AFP) levels were measured before the commencement of GLE/PIB treatment. GLE/PIB was initiated at $300 \mathrm{mg} / 120 \mathrm{mg}$ daily for 12 weeks. Patients were followed at 4-, 12- and 24-week after the initiation of GLE/PIB treatment. During each study visit, patient were monitored for complete blood counts, liver and renal biochemistry, clotting profiles and HCV RNA levels. HCV GT and RNA levels were determined by COBAS 4800 system (Roche Diagnostic, Hong Kong, China), and the lower limit for detection was $15 \mathrm{IU} / \mathrm{mL}$. Any clinically significant events or side effects were also documented. AFP was measured before commencement of treat-

Table 1. Clinical characteristics of patients with severe renal impairment and received glecaprevair/pibrentasvir treatment

\begin{tabular}{|c|c|}
\hline Patient characteristic & Value \\
\hline Sex, M/F & $12 / 9$ \\
\hline Age (years) & $62.5 \pm 7.8$ \\
\hline \multicolumn{2}{|l|}{ Severity of renal impairment } \\
\hline Pre-dialysis & 5 \\
\hline Dialysis, PD/HD & $7 / 9$ \\
\hline Duration of dialysis (months) & $33.6 \pm 38.6$ \\
\hline \multicolumn{2}{|l|}{ Cause of renal failure } \\
\hline Unknown/DM/GN/HT & $7 / 7 / 6 / 1$ \\
\hline \multicolumn{2}{|c|}{ Laboratory parameters prior to GLE/PIB treatment } \\
\hline Serum $\mathrm{Cr}(\mu \mathrm{mol} / \mathrm{L})$ & $659.8 \pm 287.8$ \\
\hline $\mathrm{eGFR}\left(\mathrm{mL} / \mathrm{min} / 1.73 \mathrm{~m}^{2}\right)$ & $12.9 \pm 6.2$ \\
\hline $\mathrm{ALT}(\mathrm{U} / \mathrm{mL})$ & $29.0 \pm 16.8$ \\
\hline AST (U/mL) & $28.9 \pm 17.0$ \\
\hline Albumin (g/L) & $34.4 \pm 6.6$ \\
\hline Bilirubin ( $\mu \mathrm{mol} / \mathrm{L})$ & $16.8 \pm 19.6$ \\
\hline PT (seconds) & $11.4 \pm 1.7$ \\
\hline INR & $1.0 \pm 0.1$ \\
\hline HCV GT, GT2/GT3/GT6 & $7 / 6 / 8$ \\
\hline HCV RNA (IU/mL) & $7.2 \times 10^{6} \pm 1.7 \times 10^{7}$ \\
\hline
\end{tabular}

Values are presented as mean \pm standard deviation or number.

$M$, male; F, female; $P D$, peritoneal dialysis; $H D$, hemodialysis; $D M$, diabetes mellitus; GN, glomerulonephritis; HT, hypertension; GLE/PIB, glecaprevir/ pibrentasvir; $\mathrm{Cr}$, creatinine; eGFR, estimated glomerular filtration rate; $\mathrm{ALT}$, alanine aminotransferase; AST, aspartate aminotransferase; PT, prothrombin time; INR, international normalized ratio; HCV, hepatitis C virus; GT, genotype. 
ment and also repeated at 24 weeks after treatment. To monitor for liver decompensation in PD patients, we also documented the number of exchanges, net ultrafiltration and residual urine volume before and after GLE/PIB treatment.

\section{Statistical analysis}

The primary outcome was the rate of sustained virological response at 12 weeks (SVR12, defined as an HCV RNA level of less than $15 \mathrm{IU} / \mathrm{mL} 12$ weeks after the end of treatment). Secondary outcomes include the rate of sustained virological response at 24 weeks (SVR24, defined as an HCV RNA level of less than $15 \mathrm{IU} / \mathrm{mL}$ 24 weeks after the end of treatment), changes in HCV RNA levels, liver and renal function and adverse events. Continuous variables were expressed as mean \pm standard deviation and analysed with
Student's $t$ test or Wilcoxon test where appropriate. Categorical variables were expressed as frequency (percentage), and analysed with chi-square test or Fisher-Exact test where appropriate. All statistical analysis were performed by statistical software SPSS (version 24; IBM, Armonk, NY, USA), and a $P$-value $<0.05$ was considered statistical significant.

\section{RESULTS}

\section{Patients}

A total of 21 patients were included for analysis (11 from Hong Kong and 10 from Taiwan) (Tables 1, 2). All patients were Asians and received GLE/PIB $300 \mathrm{mg} / 120 \mathrm{mg}$ once daily. The mean dura-

Table 2. The transient elastography scores and ultrasonographic findings of 21 patients who had severe renal impairment and received glecaprevir/ pibrentasvir treatment

\begin{tabular}{|c|c|c|}
\hline & Transient elastography scores $(\mathrm{kPa})$ & Ultrasonography findings \\
\hline Patient 1 & 18.4 & Liver cysts with mild increase in liver echogenicity \\
\hline Patient 2 & 18.2 & Moderate increase in liver echogenicity \\
\hline Patient 3 & 14.3 & Hepatosplenomegaly \\
\hline Patient 4 & 17.3 & Nodular liver and increased echogenicity \\
\hline Patient 5 & $6.0^{*}$ & Increased liver echogenicity \\
\hline Patient 6 & 27.0 & Cirrhotic liver with marked splenomegaly \\
\hline Patient 7 & 13.4 & Nodular liver, mild splenomegaly \\
\hline Patient 8 & 13.2 & Lobulated liver \\
\hline Patient 9 & 26.0 & Nodular liver \\
\hline Patient 10 & $5.2^{*}$ & Nodular liver \\
\hline Patient 11 & 12.5 & Lobulated liver with no splenomegaly \\
\hline Patient 12 & 11.0 & Increased liver echogenicity \\
\hline Patient 13 & $\mathrm{ND}^{+}$ & Increased liver echogenicity \\
\hline Patient 14 & 5.1 & Increased liver echogenicity, splenomegaly \\
\hline Patient 15 & $\mathrm{ND}^{\dagger}$ & Increased liver echogenicity with mild splenomegaly \\
\hline Patient 16 & $\mathrm{ND}^{+}$ & Nodular liver with mild splenomegaly \\
\hline Patient 17 & 39.7 & Markedly nodular liver \\
\hline Patient 18 & 11.0 & Nodular liver \\
\hline Patient 19 & $\mathrm{ND}^{+}$ & Nodular liver, post-RFA changes of HCC \\
\hline Patient 20 & $\mathrm{ND}^{+}$ & Mildly nodular liver with mild splenomegaly \\
\hline Patient 21 & 38.0 & Nodular liver with mild splenomegaly \\
\hline
\end{tabular}

ND, not done; RFA, radiofrequncy ablation; HCC, hepatocellular carcinoma.

*These two patients were treated because they have history of cryoglobulinaemia.

${ }^{\dagger}$ Fibroscan was not performed in these patients because of no reimbursement, the diagnosis of cirrhosis was based on compatible clinical/ultrasonographic features. 


\section{CLINCAL and MOLECULAP}

Volume_26 Number_4 October 2020

tion of treatment was $11.2 \pm 1.8$ weeks. Seven, six, and eight patients were infected with GT2, GT3, and GT6 HCV respectively. Two patients had history of HCV-related membranoproliferative glomerulonephritis (one received prednisolone $50 \mathrm{mg} /$ day before GLE/PIB treatment and the other patient refused HCV treatment when renal biopsy was performed 2 years before the use of GLE/ PIB). Twenty patients were treatment-naïve and one patient was treatment-experienced (previously received pegylated interferon [IFN] treatment).

\section{Virological response}

Of the 21 patients, one patient died of PD-related peritonitis during treatment and two were lost to follow up. The SVR12 in
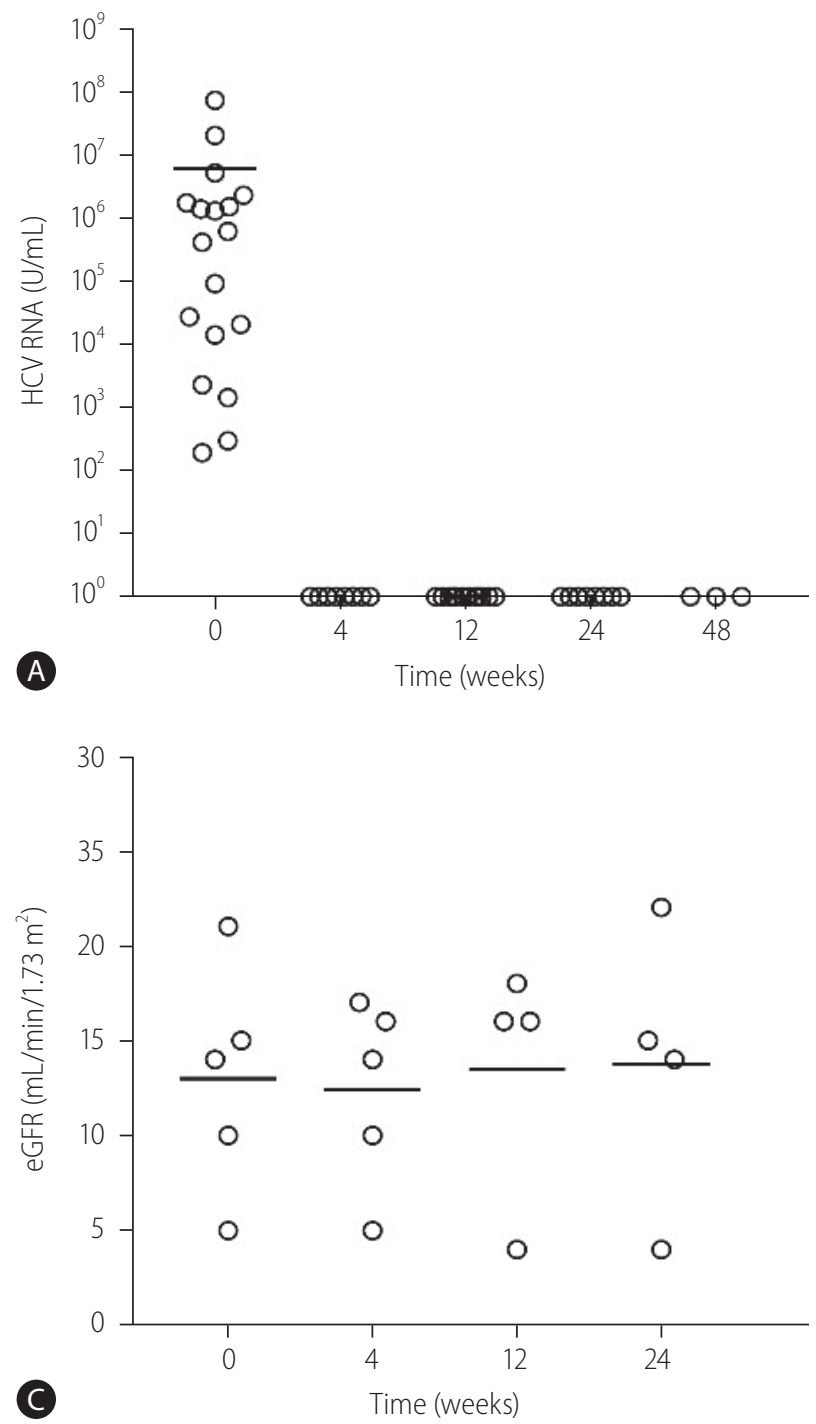

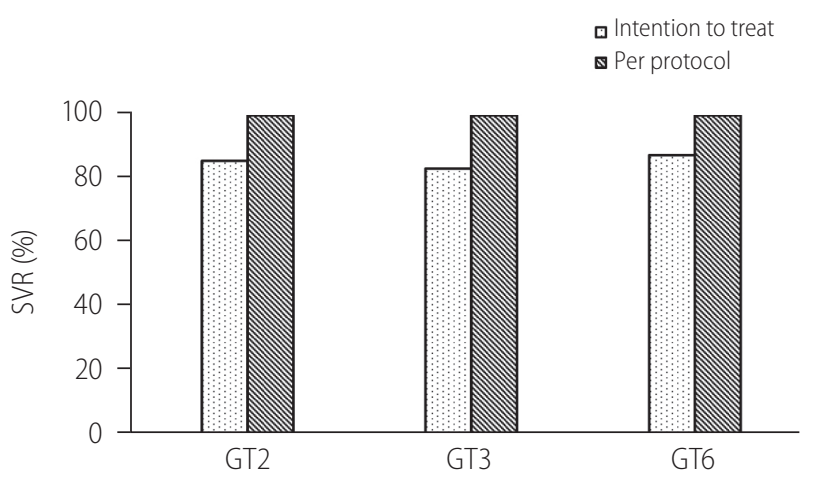

Figure 2. The rate of sustained virological response (SVR) as analysed per protocol or by intention to treat (ITT).

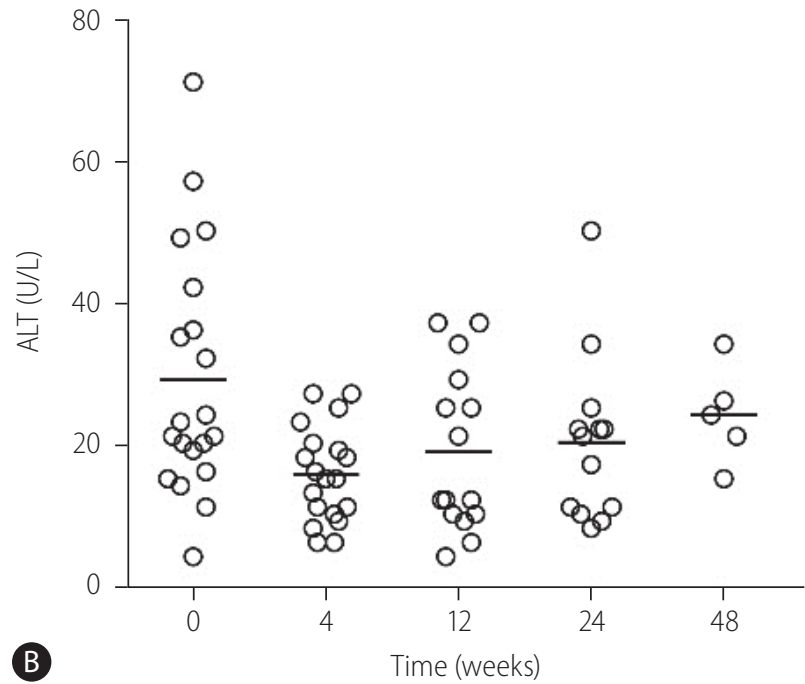

Figure 1. Changes in (A) HCV RNA, (B) ALT, and (C) renal function in patients with severe renal impairment and received glecaprevair/pibrentasvir treatment. HCV, hepatitis C virus; ALT, alanine aminotransferase; eGFR, estimated glomerular filtration rate. 
the remaining 18 patients was $100 \%$. The baseline HCV RNA level before initiation of treatment was $7.2 \times 10^{6} \pm 1.7 \times 10^{7} \mathrm{IU} / \mathrm{mL}$. All patients achieved undetectable HCV RNA at 4, 12, and 24 weeks after treatment initiation respectively (Fig. 1A). There was no difference in the SVR12 rates between GT2, GT3, and GT6 patients (100\% for all, $P>0.05$ for all comparison). There was also no difference in SVR12 rates between patients who were treatment-naïve or treatment-experienced (100\% for both, $P>0.05)$ (Fig. 2). Three patients had elevated alanine aminotransferase (ALT) at baseline $(59.3 \pm 10.7 \mathrm{U} / \mathrm{mL})$, and the ALT level decreased to $23.0 \pm 4.0 \mathrm{U} / \mathrm{mL}, 23.0 \pm 2.8 \mathrm{U} / \mathrm{mL}$ and $24.0 \pm 8.9 \mathrm{U} / \mathrm{mL}$ at 4,12 , and 24 weeks after treatment $(P=0.155,0.027$, and 0.039 compared with baseline value) (Fig. 1B). During a mean follow-up of $31.2 \pm 13.7$ weeks, there was no virological breakthrough or increase in liver transaminase levels. Nine patients had followed up to 1 year, all showed undetectable HCV RNA during their last follow-up visits and the SVR24 rate was 100\% in these nine patients.

\section{Effect on renal function}

Sixteen patients received dialysis (seven were on PD and nine were on $\mathrm{HD}$ ). None of them required intensification of dialysis regimen after initiation of GLE/PIB. In PD patients, there was no difference in the mean number of PD exchanges ( $3.2 \pm 0.4$ vs. $3.2 \pm 0.4$ exchanges/day, $P=1.00)$, volume of net ultrafiltration (780.0 \pm 204.9 vs. $720.0 \pm 192.4 \mathrm{~mL} /$ day, $P=0.180)$ and residual urine volume $(560.0 \pm 260.8$ vs. $680.0 \pm 389.9 \mathrm{~mL} /$ day, $P=0.317$ ) before and after GLE/PIB treatment. Five patients were not on dialysis before the commencement of GLE/PIB, and all showed stable renal function for up to 24 weeks after initiation of treatment (Fig. 1C).

\section{Drug tolerability and adverse events}

One patient had early discontinuation of GLE/PIB because of patient preference and was unrelated to treatment side effects, and this patient also achieved SVR12 despite receiving only 4 weeks of GLE/PIB treatment. One patient died of PD-related fungal peritonitis, which occurred at 3 months after GLE/PIB treatment. GLE/PIB treatment was well-tolerated in other patients.

\section{DISCUSSION}

Our current data showed that GLE/PIB treatment was associat- ed with high efficacy and tolerability in patients with chronic HCV infection and severe renal impairment. In this cohort, the rate of SVR12 was $100 \%$ and the suppression of HCV RNA to undetectable levels occurred as early as 4 weeks. Such excellent therapeutic response was in line with the data in general population and previous studies in patients with renal impairment. ${ }^{13-19}$ While GLE/ PIB was stated to show pan-genotypic efficacy in HCV infection, there was very limited data on some specific GT, for instance GT6. In this context, a previous study which investigated the use of GLE/PIB in patients with renal impairment only included one patient with GT6. ${ }^{19}$ In our study, we have included a considerable proportion of patients with GT6 (38.1\%), and the SVR12 rate was $100 \%$ in these patients. The high SVR rates in this cohort were in line the data from previous pivotal studies (e.g., ENDURANCE-5, 6; SURVEYOR-2 and VOYAGE-1 and -2), which include considerable number of compensated cirrhotic/non-cirrhotic GT-6 patients who did not have significant renal dysfunction. ${ }^{22-25}$ As in general population, we observed no significant difference in treatment response between patients of different $\mathrm{GT}^{18}$ There is also concern whether the therapeutic response of GLE/PIB is sustainable in patients with renal failure as they are recognized to have impaired innate and adaptive immunity. ${ }^{26}$ In this study, nine patients had follow-up data up to 1 year and the rate of SVR24 was 100\% with no virological breakthrough. The demonstration of sustainable HCV eradication had important clinical implications for infection control in dialysis units, allocation of dialysis machines and kidney transplantation. In the present cohort, patients were predominantly treatment-naïve (up to $95 \%$ ) and all showed excellent therapeutic response, while the remaining patient who was previously treated with pegylated-IFN also showed successful viral clearance after GLE/PIB treatment.

Drug tolerability and renal safety is an important concern for the use of DAA in patients with severe renal impairment. Our current data suggested that GLE/PIB treatment was associated with favourable drug tolerability in patients with advanced CKD, which was in line with a previous study in patients with severe renal dysfunction. ${ }^{19}$ In our cohort, only one patient had early discontinuation of GLE/PIB because of his own preference and yet he still achieved SVR12 despite receiving GLE/PIB for only 4 weeks. One patient died in this study due to PD-associated fungal peritonitis which was unrelated to GLE/PIB treatment. While previous studies had included predominantly HD patients and there was relatively limited data on the use of GLE/PIB in PD patients, the current guidelines recommend clinicians to follow practices in HD when treating HCV infection in PD patients. ${ }^{16,19}$ Notwithstanding, phar- 
macokinetics and drug elimination are different in PD and HD patients. ${ }^{27}$ Our current data, which included a significant proportion (33.3\%) of PD patients, suggested that GLE/PIB was also effective and well tolerated in PD patients. The detection and monitoring of liver decompensation (e.g., increase in ascites) can be difficult in PD patients due to the presence of PD dialysate. Our present showed that there was no change in the number of exchanges, net ultrafiltration and residual urine volume in PD patients before and after GLE/PIB treatment. Such data is important as PD is a growing modality of renal replacement therapy in many localities, especially in developing countries and the Asia-Pacific region where HCV infection is still prevalent. ${ }^{28,29}$ For patients who were not on dialysis, their renal function showed no significant deterioration during the course of treatment and had remained stable for 24 weeks.

GLE/PIB treatment was associated with high efficacy and tolerability in HCV-infected patients with severe renal impairment.

\section{Authors' contribution}

Yap DYH: conception and conduction of study, analysis of data and preparation of manuscript

Liu KSH, Wong GLH, Tsai MC, Chen CH, Hsu CS, Hui YT, Li MKK, Liu CH, Kan YM, Yu ML: recruitment and care of patient, preparation of manuscript

Yuen MF: Conception and conduction of study, data analysis and preparation of manuscript

\section{Conflicts of Interest}

The authors have no conflicts to disclose.

\section{REFERENCES}

1. Fabrizi F, Martin P, Messa P. New treatment for hepatitis $C$ in chronic kidney disease, dialysis, and transplant. Kidney Int 2016;89:988994.

2. Fabrizi F, Dixit V, Messa P. Impact of hepatitis $C$ on survival in dialysis patients: a link with cardiovascular mortality? J Viral Hepat 2012;19:601-607.

3. Fabrizi F, Martin P, Dixit V, Messa P. Meta-analysis of observational studies: hepatitis $\mathrm{C}$ and survival after renal transplant. J Viral Hepat 2014;21:314-324.

4. Fabrizi F, Dixit V, Messa P, Martin P. Antiviral therapy (pegylated interferon and ribavirin) of hepatitis $C$ in dialysis patients: metaanalysis of clinical studies. J Viral Hepat 2014;21:681-689.
5. Feld JJ, Jacobson IM, Hézode C, Asselah T, Ruane PJ, Gruener N, et al. Sofosbuvir and velpatasvir for HCV genotype 1, 2, 4, 5, and 6 infection. N Engl J Med 2015;373:2599-2607.

6. Foster GR, Afdhal N, Roberts SK, Bräu N, Gane EJ, Pianko S, et al. Sofosbuvir and velpatasvir for HCV genotype 2 and 3 infection. N Engl J Med 2015;373:2608-2617.

7. Curry MP, O'Leary JG, Bzowej N, Muir AJ, Korenblat KM, Fenkel JM, et al. Sofosbuvir and velpatasvir for HCV in patients with decompensated cirrhosis. N Engl J Med 2015;373:2618-2628.

8. Dumortier J, Guillaud O, Gagnieu MC, Janbon B, Juillard L, Morelon E, et al. Anti-viral triple therapy with telaprevir in haemodialysed HCV patients: is it feasible? J Clin Virol 2013;56:146-149.

9. Wiegand J, Maasoumy B, Buggisch P, Buslau A, Schiefke I, Berg T, et al. Letter: telaprevir triple therapy in chronic hepatitis $C$ genotype 1 patients receiving haemodialysis. Aliment Pharmacol Ther 2014;39:1342-1344.

10. Kaya S, Aksoz S, Baysal B, Ay N, Danis R. Evaluation of telaprevircontaining triple therapy in the treatment of chronic hepatitis $C$ in hemodialysed patients. Infect Dis (Lond) 2015;47:658-661.

11. Mehawej M, Rostaing L, Alric L, Del Bello A, Izopet J, Kamar N. Boceprevir-based triple antiviral therapy for chronic hepatitis $C$ virus infection in kidney-transplant candidates. J Transplant 2015;2015:159795.

12. Ng TI, Krishnan P, Pilot-Matias T, Kati W, Schnell G, Beyer J, et al. In vitro antiviral activity and resistance profile of the next-generation hepatitis C virus NS5A inhibitor pibrentasvir. Antimicrob Agents Chemother 2017;61:e02558-16.

13. Poordad F, Felizarta F, Asatryan A, Sulkowski MS, Reindollar RW, Landis CS, et al. Glecaprevir and pibrentasvir for 12 weeks for hepatitis C virus genotype 1 infection and prior direct-acting antiviral treatment. Hepatology 2017;66:389-397.

14. D’Ambrosio R, Pasulo L, Puoti M, Vinci M, Schiavini M, Lazzaroni S, et al. Real-world effectiveness and safety of glecaprevir/pibrentasvir in 723 patients with chronic hepatitis C. J Hepatol 2019;70:379-387.

15. Zeuzem S, Foster GR, Wang S, Asatryan A, Gane E, Feld JJ, et al. Glecaprevir-pibrentasvir for 8 or 12 weeks in HCV genotype 1 or 3 infection. N Engl J Med 2018;378:354-369.

16. Kidney Disease: Improving Global Outcomes (KDIGO) Hepatitis C Work Group. KDIGO 2018 clinical practice guideline for the prevention, diagnosis, evaluation, and treatment of hepatitis $\mathrm{C}$ in chronic kidney disease. Kidney Int Suppl (2011) 2018;8:91-165.

17. Flamm S, Mutimer D, Asatryan A, Wang S, Rockstroh J, Horsmans $Y$, et al. Glecaprevir/pibrentasvir in patients with chronic HCV genotype 3 infection: an integrated phase 2/3 analysis. J Viral Hepat 2019;26:337-349.

18. Puoti M, Foster GR, Wang S, Mutimer D, Gane E, Moreno C, et al. High SVR12 with 8-week and 12-week glecaprevir/pibrentasvir therapy: an integrated analysis of HCV genotype 1-6 patients without cirrhosis. J Hepatol 2018;69:293-300. 
19. Gane E, Lawitz E, Pugatch D, Papatheodoridis G, Bräu N, Brown A, et al. Glecaprevir and pibrentasvir in patients with $\mathrm{HCV}$ and severe renal impairment. N Engl J Med 2017;377:1448-1455.

20. Prescott LE, Simmonds P, Lai CL, Chan NK, Pike I, Yap PL, et al. Detection and clinical features of hepatitis C virus type 6 infections in blood donors from Hong Kong. J Med Virol 1996;50:168-175.

21. Thong VD, Akkarathamrongsin S, Poovorawan K, Tangkijvanich $P$, Poovorawan Y. Hepatitis C virus genotype 6: virology, epidemiology, genetic variation and clinical implication. World J Gastroenterol 2014;20:2927-2940.

22. Asselah T, Lee SS, Yao BB, Nguyen T, Wong F, Mahomed A, et al. Efficacy and safety of glecaprevir/pibrentasvir in patients with chronic hepatitis C virus genotype 5 or 6 infection (ENDURANCE-5,6): an open-label, multicentre, phase 3b trial. Lancet Gastroenterol Hepatol 2019;4:45-51.

23. Kwo PY, Poordad F, Asatryan A, Wang S, Wyles DL, Hassanein T, et al. Glecaprevir and pibrentasvir yield high response rates in patients with HCV genotype 1-6 without cirrhosis. J Hepatol 2017;67:263271.

24. National Institutes of Health (NIH). A study to evaluate the efficacy and safety of glecaprevir/pibrentasvir (ABT-493/ABT-530) in treatment-naive and treatment-experienced, non-cirrhotic asian adults with chronic hepatitis C virus genotype (GT) 1 to GT6 infection with or without human immunodeficiency virus co-infection (VOYAGE-1). NIH web site, <https://www.clinicaltrials.gov/ct2/show/results/ NCT03222583>. Accessed 13 Aug 2020.

25. National Institutes of Health (NIH). Efficacy and safety of glecaprevir/ pibrentasvir (ABT-493/ABT-530) in treatment-naive and treatmentexperienced asian adults with chronic hepatitis C virus genotype (GT) 1 to GT6 infection with compensated cirrhosis and with or without human immunodeficiency virus co-infection (VOYAGE-2). NIH web site, <https://www.clinicaltrials.gov/ct2/show/NCT03235349>. Accessed 13 Aug 2020.

26. Kato S, Chmielewski M, Honda H, Pecoits-Filho R, Matsuo S, Yuzawa $Y$, et al. Aspects of immune dysfunction in end-stage renal disease. Clin J Am Soc Nephrol 2008;3:1526-1533.

27. Smyth B, Jones C, Saunders J. Prescribing for patients on dialysis. Aust Prescr 2016:39:21-24.

28. Kwong VW, Li PK. Peritoneal dialysis in Asia. Kidney Dis (Basel) 2015;1:147-156.

29. Lim SG, Aghemo A, Chen PJ, Dan YY, Gane E, Gani R, et al. Management of hepatitis $C$ virus infection in the Asia-Pacific region: an update. Lancet Gastroenterol Hepatol. 2017;2:52-62. 